ARTICLE

Received 18 May 2015 | Accepted 1 Sep 2015 | Published 5 Oct $2015 \quad$ DOl: 10.1038/ncomms9540 OPEN

\title{
Dynamic acousto-optic control of a strongly coupled photonic molecule
}

\author{
Stephan Kapfinger ${ }^{1,2}$, Thorsten Reichert2,3, Stefan Lichtmannecker ${ }^{2,3}$, Kai Müller 2,3,4, Jonathan J. Finley 2,3,
} Achim Wixforth ${ }^{1,2,5}$, Michael Kaniber ${ }^{3}$ \& Hubert J. Krenner ${ }^{1,2,5}$

Strongly confined photonic modes can couple to quantum emitters and mechanical excitations. To harness the full potential in quantum photonic circuits, interactions between different constituents have to be precisely and dynamically controlled. Here, a prototypical coupled element, a photonic molecule defined in a photonic crystal membrane, is controlled by a radio frequency surface acoustic wave. The sound wave is tailored to deliberately switch on and off the bond of the photonic molecule on sub-nanosecond timescales. In time-resolved experiments, the acousto-optically controllable coupling is directly observed as clear anticrossings between the two nanophotonic modes. The coupling strength is determined directly from the experimental data. Both the time dependence of the tuning and the inter-cavity coupling strength are found to be in excellent agreement with numerical calculations. The demonstrated mechanical technique can be directly applied for dynamic quantum gate operations in state-of-the-art-coupled nanophotonic, quantum cavity electrodynamic and optomechanical systems.

\footnotetext{
${ }^{1}$ Lehrstuhl für Experimentalphysik 1 and Augsburg Centre for Innovative Technologies (ACIT), Universität Augsburg, Universitätsstrasse 1, 86159 Augsburg, Germany. ${ }^{2}$ Nanosystems Initiative Munich (NIM), Schellingstrasse 4, 80799 München, Germany. ${ }^{3}$ Walter Schottky Institut and Physik Department, Technische Universität München, Am Coulombwall 4, 85748 Garching, Germany. ${ }^{4}$ E. L. Ginzton Laboratory, Stanford University, Stanford, California 94305 , USA. ${ }^{5}$ Center for NanoScience (CeNS), Ludwig-Maximilians-Universität München, Schellingstrasse 4, 80799 München, Germany. Correspondence and requests for materials should be addressed to H.J.K. (email: hubert.krenner@physik.uni-augsburg.de).
} 
T wo-dimensional photonic crystal membranes provide a versatile planar architecture for integrated photonics to control the propagation of light on a chip using highquality optical cavities, waveguides, beamsplitters or dispersive elements ${ }^{1}$. When combined with highly nonlinear quantum emitters, quantum photonic networks ${ }^{2,3}$ operating at the singlephoton level ${ }^{4}$ come within reach. Towards large-scale quantum photonic networks ${ }^{5,6}$, selective dynamic control of individual components and deterministic interactions between different constituents are of paramount importance ${ }^{7}$. This indeed calls for switching speeds ultimately on the system's native timescales to enable Landau-Zener non-adiabatic control schemes, ${ }^{8,9}$. For example, manipulation via electric fields or all-optical means have been used for switching in nanophotonic circuits ${ }^{10,11}$ and cavity quantum electrodynamics studies ${ }^{12-14}$.

Here, we demonstrate dynamic control of the coherent interaction between two coupled photonic crystal (PhC) nanocavities forming a photonic molecule $(\mathrm{PM})^{15-17}$. By using an electrically generated radio frequency $(\mathrm{RF})$ surface acoustic wave (SAW), we achieve optomechanical tuning ${ }^{18}$. SAWs have been used to dynamically control nanophotonic ${ }^{18-20}$, plasmonic $^{21-23}$, integrated optical devices ${ }^{24,25}$ and quantum dot (QD) emitters $^{26-29}$ at frequencies up to several GHz. The tuning range of our method is sufficiently large to compensate for the inherent fabrication-related cavity mode detuning and the operation speed exceeds that of typical ${ }^{30}$ and downscaled ${ }^{31}$ resonant mechanical approaches. Our findings open a route towards nanomechanically gated protocols ${ }^{9}$, which hitherto have inhibited the realization in all-optical schemes ${ }^{32,33}$. Moreover, the mechanical nature of the SAW makes them ideally suited to generate tailored classical phonon fields ${ }^{34}$. The $\mathrm{GHz}$ frequencies accessible by SAWs perfectly match that of the mechanical modes in membrane-type PhC photonic ${ }^{35}$ and optomechanical cavities ${ }^{36}$. Thus, our results pave the way towards native mechanical control in these scaled on-chip optomechanical systems.

\section{Results}

Implementation of acoustically tunable PM. The on-chip PM studied here consists of two L3-type missing hole cavities ${ }^{37}$, labelled $C_{1}$ and $C_{2}$, defined in a two-dimensional PhC membrane. Its static coupling strength $J$ is known to depend exponentially on the separation between the two cavities ${ }^{17}$. In our sample, the cavities are offset symmetrically by $d=5$ lattice constants $(a=260 \mathrm{~nm})$ along each primitive direction of the PhC lattice (Supplementary Fig. 1), as can be seen in the scanning electron micrograph in Fig. 1a. The finite coupling strength $J$ leads to the formation of two normal modes; a bonding mode $M_{-}$and an antibonding mode $M_{+}$. Note that the mode indices in $M_{ \pm}$refer to the respective normal mode frequencies $\omega \pm$, and thus are opposite to the spatial symmetry of the mode. In the most general case, the two cavities $C_{1}$ and $C_{2}$ exhibit resonance frequencies $\omega_{1}$ and $\omega_{2}$, split by a finite detuning $\Delta=\omega_{2}-\omega_{1}$. Thus, the normal mode frequencies can be expressed by:

$$
\omega_{ \pm}=\omega_{0} \pm \frac{1}{2} \sqrt{\Delta^{2}+J^{2}}
$$

with $\omega_{0}=\left(\omega_{1}+\omega_{2}\right) / 2$ being the centre frequency. In Fig. 1b, we present two emission spectra (see Methods section) recorded for spatially exciting either $C_{1}$ (blue) or $C_{2}$ (red) of a typical PM, denoted as PM1. For PM1, each cavity exhibits a distinct single mode, split by $\omega_{ \pm}=\omega_{0} \pm \frac{1}{2} \sqrt{\Delta_{0}^{2}+J^{2}}$, with $\Delta_{0}$ being the static detuning. Such distinct and localized mode patterns are found for the majority of the PMs. This suggests that $\Delta_{0}>J$, and that the inter-cavity coupling is strongly suppressed. Therefore, as- fabricated and nominally identical cavities are only weakly interacting due to $\Delta_{0} \neq 0$, which arises from inevitable imperfections during nanofabrication. To overcome this fundamental limitation and achieve dynamic control of the individual cavity resonances, we use an optomechanical approach using RF SAWs ${ }^{18,19}$. As indicated in the schematic illustration of Fig. 1a, these quasi-monochromatic acoustic phonons are generated by an interdigital transducer (IDT; see Methods section), propagate along the $x$ axis of the PM and dynamically deform the individual cavities. The amplitude of this mechanism, $\hbar A>1 \mathrm{meV}$, exceeds both the static detuning, $\Delta_{0}$, and the coupling strength, $J$, which enables us to dynamically tune the PM completely in and out of resonance. For our chosen geometric arrangement, the cavities are separated by a distance $\Delta x$ along the SAW propagation direction, the modulations of $\omega_{1}$ and $\omega_{2}$ are phase shifted (in unit radians) by $\phi_{12}=\Delta x \times \omega_{\mathrm{SAW}} / c_{\mathrm{SAW}}$, with $\omega_{\text {SAW }}$ and $c_{\text {SAW }}$ being the SAW angular frequency and speed of sound, respectively. We deliberately set the acoustic wavelength, $\lambda_{\text {SAW }}$, such that it is commensurate with the spatial separation of the two cavity centres $2 \Delta x=\lambda_{\text {SAW }}$. As illustrated in the schematic in Fig. $1 c$, for the selected phase, the maximum (minimum) of the SAW expands $C_{1}$ (compresses $C_{2}$ ), which in turn gives rise to a red (blue) shift of their respective resonances ${ }^{18}$. The propagation of the sound wave along the $x$ axis of the PM leads to a timedependent detuning of the two cavities

$$
\Delta(t)=\Delta_{0}+\Delta_{\bmod } \sin \left(\omega_{\mathrm{SAW}} t\right) .
$$

The amplitude of this modulation, $\Delta_{\bmod }=$ $\sqrt{A_{1}^{2}+A_{2}^{2}-2 A_{1} A_{2} \cos \left(\phi_{12}\right)}$, depends on the amplitudes of the modulations of the individual cavities, $A_{1}$ and $A_{2}$, and is maximum for $\phi_{12}=180^{\circ}$. For $\Delta_{\bmod }>\Delta_{0}$, the detuning passes through zero, which results according to equation (1) in an avoided crossing of the normal modes. In Fig. 1d, the resulting temporal evolutions of the normalized mode frequencies $\left(\omega-\omega_{0}\right) / J$ obtained using equations (1) and (2) are evaluated over one acoustic cycle of $T_{\text {SAW }}$ for a typical set of experimentally achievable parameters $\left(J=1.2 \Delta_{0}, A_{1}=1.5 \Delta_{0}, A_{2}=1.9 \Delta_{0}\right.$ and $\phi_{12}=162^{\circ}$ ). The dashed lines show the time evolution of the non-interacting $(J=0)$ modes $M_{1}$ (blue) and $M_{2}$ (red). In strong contrast, the normal modes $M_{+}$and $M_{-}$start with initially $M_{1^{-}}$ like (blue) and $M_{2}$-like (red) single-cavity character, and develop to fully mixed symmetric (bonding) and antisymmetric (antibonding) character (green) at resonance. At this point, the coupling strength $J$ of the PM can be deduced directly from the splitting of the avoided crossing. After traversing through the avoided crossing, the initial character of the modes is exchanged, with $M_{+}$and $M_{-}$possessing $M_{2}$-like (blue) and $M_{1}$-like character, respectively. Over the duration of one full acoustic cycle, the two modes are brought into resonance at two distinct times, giving rise to two avoided crossings, restoring the initial configuration. We performed full finite difference time-domain (FDTD) simulations to confirm and, in particular, quantitate the dynamic coupling. The evaluation for the used sample geometry is shown in Supplementary Fig. 2. We evaluate the calculated profiles of the electric field component in the plane of the $\mathrm{PhC}$ membrane and perpendicular to the SAW propagation $\left(E_{y}\right)$ in Fig. 1e. In the two left panels, the detuning is set to three times the coupling strength $(\Delta=3 J)$, and thus the two modes remain well localized in one of the two cavities. For the resonance case, $\Delta=0$, shown in the two right panels of Fig. 1e, the $E_{y}$ exhibit the characteristic symmetric and antisymmetric superpositions for the $M_{-}$and $M_{+}$modes, respectively. For our sample geometry, the FDTD simulations predict $\hbar J_{\text {sim }}=0.71 \mathrm{meV}$. This value is set by the chosen separation, that is, barrier thickness, between the two cavities. 
a

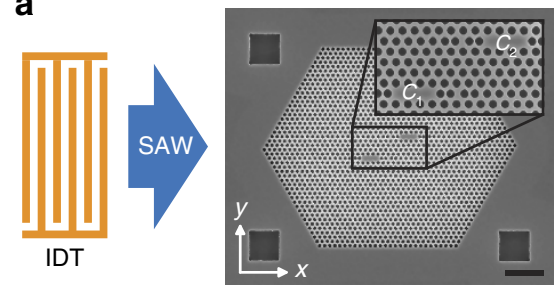

C

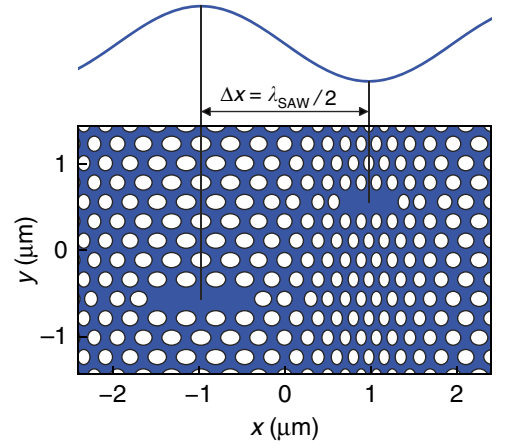

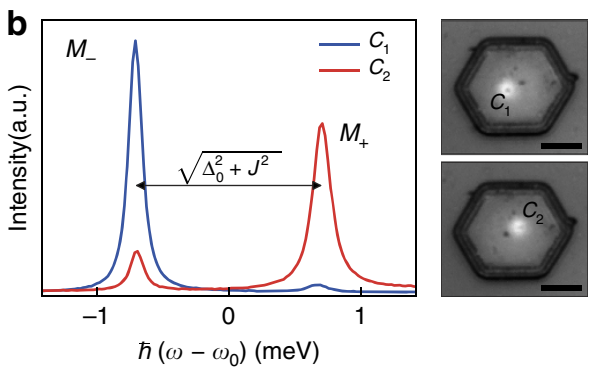

d

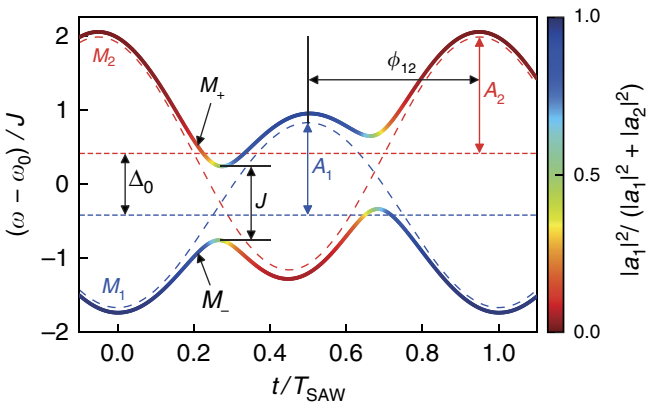

e
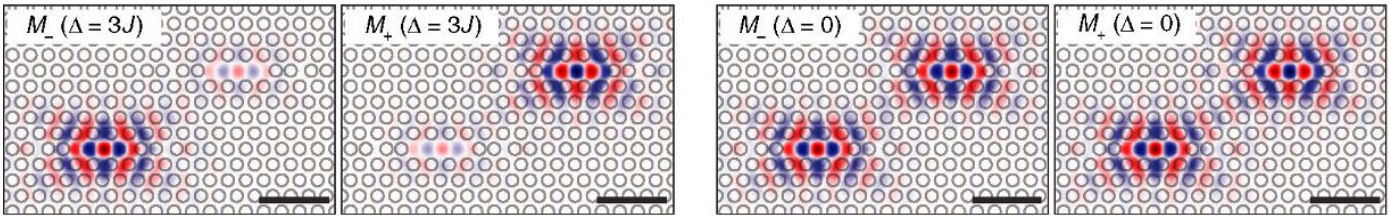

Figure 1 | PM with acousto-optic tuning mechanism. (a) Scanning electron micrograph of the PM and schematic of the device layout. The IDT launches a SAW that propagates across the PM. Scale bar, $2 \mu \mathrm{m}$ (IDT not to scale). (b) PL spectra showing the two fundamental modes of the PM and optical microscope images of the excitation laser spot. The blue and the red curves were obtained by exciting on cavity $C_{1}$ and $C_{2}$, respectively. Owing to a finite static detuning $\Delta_{0}>J$, the modes are confined within their respective cavities. The centre energy is $\hbar \omega_{0}=1.260 \mathrm{eV}$. The cavities can be spatially resolved in our optical set-up, allowing selective excitation of the individual cavities. Scale bars, $5 \mu \mathrm{m}$. (c) Acousto-optic tuning. The resonance frequencies of the cavities are modulated by the SAW-induced strain field (amplitude exaggerated). Setting half the SAW wavelength equal to the cavity separation, the cavities can be tuned relative to each other, giving rise to a time-dependent detuning $\Delta(t)=\Delta_{0}+\Delta_{\bmod } \sin \left(\omega_{\mathrm{SAW}} t\right)$. (d) Evolution of the modes over one SAW period. The dashed lines represent the single-cavity modes $M_{1}$ and $M_{2}$, and the solid lines represent the normal modes $M_{+}$and $M_{-}$. The colour scale corresponds to the decomposition into single-cavity modes, with green denoting the symmetric and antisymmetric superposition on resonance. The static detuning $\Delta_{0}$ and the coupling strength $J$ are intrinsic parameters of the PM. The tuning amplitudes $A_{1,2}$ and the phase shift $\phi_{12}$ are determined by the amplitude and wavelength of the SAW, respectively. (e) FDTD simulations of the mode profiles $\left(E_{y}\right)$. For the case of detuned cavities $(\Delta=3 J)$, the modes are localized within the individual cavities. On resonance $(\Delta=0)$, one obtains the symmetric (bonding) and the antisymmetric (antibonding) mode. Scale bar, $1 \mu \mathrm{m}$.

Direct observation of SAW-controlled inter-cavity coupling. To experimentally verify the dynamic control of a PM by a $\omega_{\mathrm{SAW}} / 2 \pi=800 \mathrm{MHz}$ SAW, we performed time-resolved spectroscopy with optical excitation at a well-defined time during the acoustic cycle. The emission of the PM was analysed in the time domain and as a function of relative emission frequency $\omega-\omega_{0}$. We measured time-dependent emission spectra of the two cavities of PM1 for three characteristic modulation amplitudes. For a weak modulation, $\Delta_{\text {mod }}<\Delta_{0}$, shown in Fig. 2 a, $C_{1}$ and $C_{2}$ show the expected phase-shifted sinusoidal spectral modulations centred (dashed horizontal lines) at their unperturbed resonances, which decay with a characteristic time constant of $\sim 1.3 \mathrm{~ns}$. As the modulation is increased to $\Delta_{\text {mod }}=\Delta_{0}$ (Fig. 2b), the two single-cavity modes are brought into resonance at one distinct time $t_{0}=0.6 \mathrm{~ns}$ during the acoustic cycle. At this time, coherent coupling leads to the formation of bonding and antibonding normal modes. This directly manifests itself in the experimental data due to the emergence of new emission features stemming from the spatial delocalization of the bonding and antibonding modes. For the initially lower frequency cavity $\left(C_{1}\right)$, a new signal appears at the frequency of the normal mode $M_{+}$, which was initially confined within the other cavity $\left(C_{2}\right)$. The initially higher-frequency cavity $\left(C_{2}\right)$ exhibits precisely the opposite behaviour, with the normal mode $M_{-}$appearing at time $t_{0}$. The normal modes are split by the coupling strength $\hbar J=(0.68 \pm 0.04) \mathrm{meV}$, very close to the value expected from our FDTD simulations. For further increased detuning $\Delta_{\bmod }>\Delta_{0}$, the two modes are brought into resonance at two distinct times, $t_{1}$ and $t_{2}$, during the acoustic cycle. After the first resonance at $t_{1}$, coupling is suppressed, $\Delta>J$, both modes are effectively decoupled and their single-cavity characters are exchanged compared with the initial configuration. The lower frequency mode $M_{-}$, which is initially $C_{1}$-like, is switched to $C_{2}$-like character after $t_{1}$, and vice versa. At the second resonance at $t_{2}$, the system is reverted to its original configuration at the beginning of the acoustic cycle. This sequence of two time-offset coupling events gives rise to the experimentally observed anticorrelated time evolutions of the emission of $C_{1}$ and $C_{2}$. We extracted the time-dependent emission frequencies detected from the two cavities of PM1 and plotted them ( $C_{1}$ circles, $C_{2}$ triangles $)$ for the three modulation amplitudes in Fig. 2d-f.

From the set of measurements with varying tuning amplitude, we obtain mean values for the free parameters of the model, $\hbar\langle J\rangle_{\mathrm{PM} 1}=(0.70 \pm 0.04) \mathrm{meV},\left\langle A_{1} / A_{2}\right\rangle_{\mathrm{PM} 1}=0.75 \pm 0.05$ and 
a
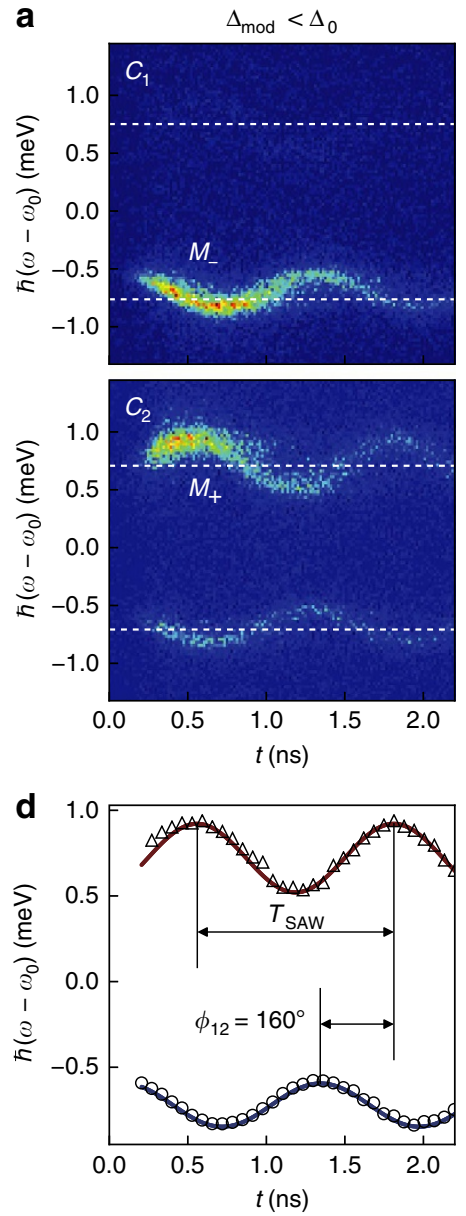

b
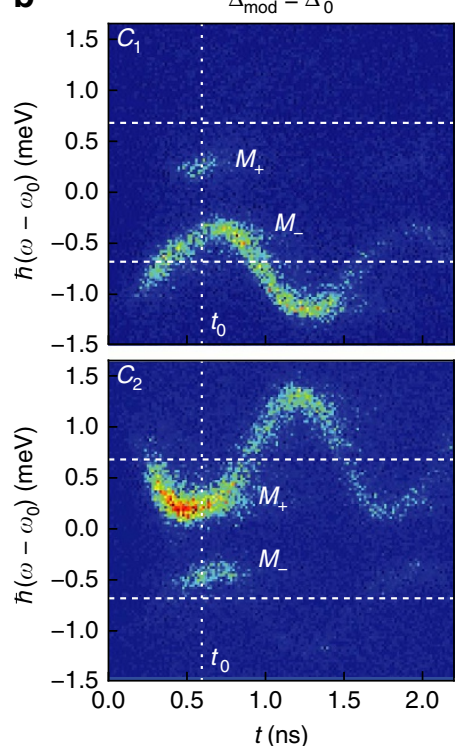

e

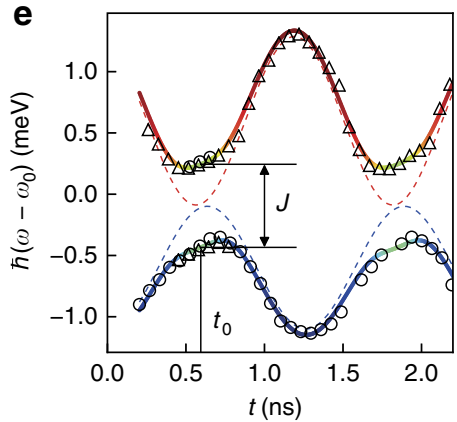

C
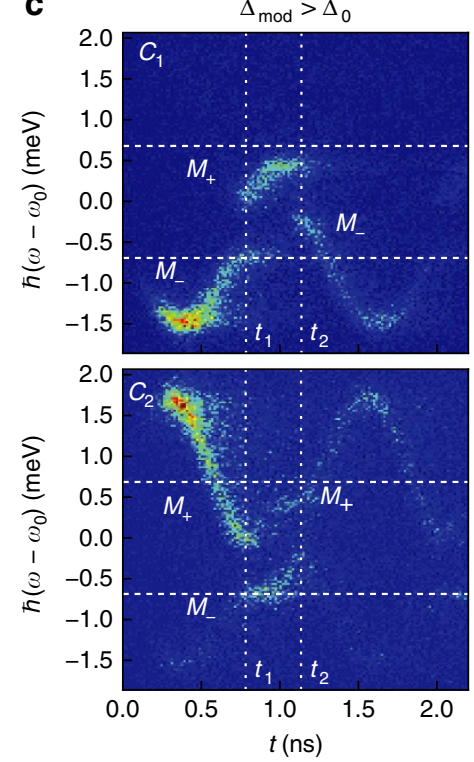

$\mathbf{f}$

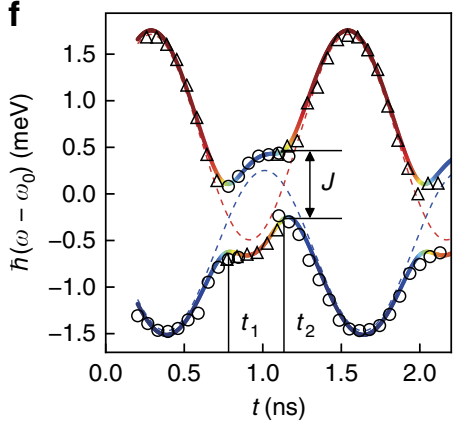

Figure 2 | Experimental characterization for three different modulation amplitudes. (a-c) Time-resolved PL maps, measured on cavity $C_{1}$ (upper panels) and $C_{2}$ (lower panels). (d-f) Mode frequencies extracted from the measurements on $C_{1}$ (circles) and $C_{2}$ (triangles), fitted with the coupled mode model. For $\Delta_{\text {mod }}<\Delta_{0}(\mathbf{a}, \mathbf{d})$, the modes are effectively decoupled. Their frequencies are modulated sinusoidally by the SAW with a phase difference of $\phi_{12}=160^{\circ}$. For $\Delta_{\text {mod }}=\Delta_{0}(\mathbf{b}, \mathbf{e})$, there is a time $t_{0}$ at which the cavities are in resonance. At this point, the modes are split by the coupling strength $J$ and they are delocalized over the PM, thus, both modes can be observed simultaneously on each cavity. When $\Delta_{\bmod }>\Delta_{0}$ (c,f), the modes exhibit an avoided crossing. This happens twice in each SAW cycle at times $t_{1}$ and $t_{2}$.

$\left\langle\phi_{12}\right\rangle_{\mathrm{PM} 1}=(155 \pm 10)^{\circ}$. Moreover, we note that the observed phase shift unambiguously confirms efficient coupling of the SAW into the sub- $\lambda_{\text {SAW }}$ membrane. Its small deviation from the ideal value of $180^{\circ}$ arises from the finite difference in the phase velocity of the acoustic wave within the membrane and the region of the transducer. The results from this model are plotted as lines and the character of the mode are colour coded. Clearly, our experimental data are in excellent agreement with the normal mode model detailed in the Methods section for all three modulation amplitudes. Both the time-dependent spectral modulation as well as the switching of the character of the modes are nicely reproduced.

Statistical analysis and comparison with FDTD simulation. Such behaviour was experimentally confirmed for five different, nominally identical PMs for which we evaluated the mean of their respective key parameters. The experimentally observed static detuning $\Delta_{0}$ and coupling strength $J$ are summarized in Fig. 3a. While the coupling strength, $\hbar J=(0.8 \pm 0.1) \mathrm{meV}$, (blue) does not vary from PM to PM, the values of the static detuning, $\Delta_{0}$, shows a pronounced scatter ranging between -1.2 and $+3.7 \mathrm{meV}$. These observations are in fact expected. $J$ exponentially depends on the symmetric inter-cavity offset, $d$, and is thus robust and insensitive to small deviations from the ideal geometry
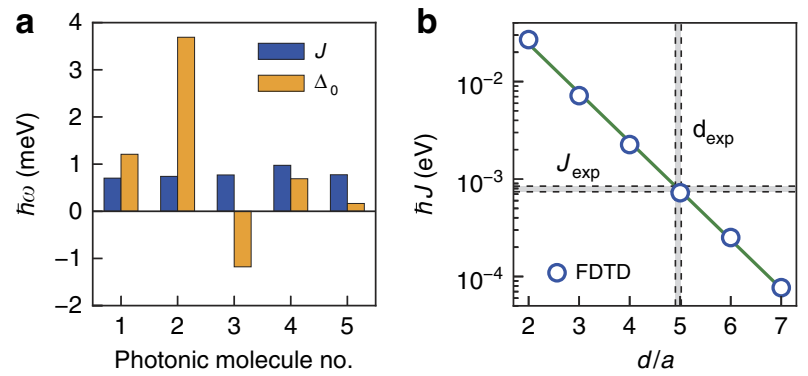

Figure 3 | Statistical variation of PM properties and comparison with FDTD simulation. (a) Coupling strength $J$ and static detuning $\Delta_{0}$ measured on five nominally identical PMs. (b) FDTD simulation of the coupling strength for different cavity separations $d$. The experimentally determined coupling strength is accurately reproduced by the simulation.

due to fabrication imperfections. In strong contrast, the absolute resonance frequencies of the two cavities forming the PM are highly sensitive to these inherent and inevitable deviations from the nominal geometry. The resulting fluctuations of the cavity resonances reflect themselves in the observed variation of $\Delta_{0}$. Indeed, we observe pronounced static coupling $\left(|J| \gg\left|\Delta_{0}\right|\right)$ only 
for one single as-fabricated PM, labelled PM5. The corresponding experimental data are presented in Supplementary Note 1 and Supplementary Figs 3 and 4 . The exponential dependence of $J$ as a function of $d$ is nicely confirmed by a best fit (line) to values calculated by FDTD (symbols) presented in Fig. 3b. The experimentally observed distribution of $J_{\exp }$ and the inter-cavity separation $d_{\text {exp }}$ derived from $J_{\exp }$ and the FDTD simulation are indicated by the shaded horizontal and vertical bars. Clearly, the measured $J_{\exp }$ and its derived $d_{\exp }$ match perfectly the calculated value of $\hbar J_{\mathrm{sim}}=0.71 \mathrm{meV}$ and the nominally set $d=5 a$. These narrow distributions centred around the calculated and nominal values are expected since $d$ is large compared with typical imperfections in the nanofabrication.

\section{Discussion}

In summary, we demonstrated dynamic optomechanical control of coherent interactions in a prototypical coupled nanophotonic system. When combined with optical nonlinearities, our tunable PM paves the way to dynamically controlled high-fidelity entanglement generation ${ }^{38}$ and distribution on a chip ${ }^{39,40}$. For larger switching rates, as required for Landau-Zener transitionbased gates ${ }^{9}$, the underlying optomechanical coupling could be enhanced further by direct antiphased SAW excitation of localized vibronic modes of either nanocavity ${ }^{35}$. Since SAWs represent nanomechanical waves, Fourier synthesis allows to shape waveforms ${ }^{34}$. This paradigm in turn can be used to deliberately speed up or slow down switching as required for static or dynamic control schemes. Moreover, SAWs or SAW waveforms can be interfered generating one- or two-dimensional nanomechanical strain fields ${ }^{41}$. Thus, our approach could be directly scaled up to large arrays of coupled cavities ${ }^{42,43}$ or waveguides ${ }^{44}$. The coherent phononic nature of SAWs was verified by Metcalfe et al. ${ }^{27}$ who demonstrated resolved sidebands in the emission of a SAW-driven QD and performed optomechanical cooling and heating in this system. For the GaAs-based nanocavities, the cavity linewidth exceeds the sideband splitting set by $\omega_{\mathrm{SAW}}$. Our approach can be readily transferred to silicon (Si)-based PhC membranes and optomechanical crystals using piezoelectric coupling layers ${ }^{45}$. On this platform, cavity photon loss rates of $<1 \mathrm{GHz}$ have been achieved $^{11}$. Therefore, our approach is ideally suited to perform optomechanically driven all-photonic Landau-Zener-based entangling quantum gates with high fidelity, even for moderate SAW frequencies. The performance of this type of quantum gates can be drastically increased using higher SAW frequencies as demonstrated for instance by Tadesse and Li (ref. 25) and weaker cavity coupling rates. The exponential dependence of the coupling strength shown in Fig. 3b predicts a decrease from $J \sim 170$ to $J<20 \mathrm{GHz}$ when the offset is increased from $5 a$ to $7 a$. This value of $J$ is indeed compatible with state-of-the-art IDTs on $\mathrm{Si}$ (ref. 46). We note that our method is compatible with superconducting two-level systems and circuit electrodynamics. In particular, the geometry of transmon-type superconducting qubits matches that of IDTs. In a recent experiment by Gustafsson et al., these highly coherent two-level systems have been strongly coupled to single SAW quanta ${ }^{47}$. Finally we note that resonant frequencies of vibronic modes in optomechanical crystals $^{36,48}$ match well with the frequency band covered by SAWs. The tight confinement of photonic and vibronic excitations in these structures gives rise to large optomechanical couplings $^{49}$. In recent theoretical work, Ludwig and Marquart ${ }^{50}$ show a rich phase diagram for arrays of such optomechanical cavities, which delicately depends on the inter-cavity coupling. Thus, our method provides a tantalizing avenue to deliberately switch on and off the interaction or drive such systems allmechanically.

\section{Methods}

Optical spectroscopy. For photoluminescence spectroscopy of the PM, the sample is cooled to $T=5 \mathrm{~K}$ in a Helium-flow cryostat with custom-built-integrated $\mathrm{RF}$ connections. Off-resonant QDs are excited by an externally triggered diode laser emitting $\sim 90$ ps pulses at a wavelength of $850 \mathrm{~nm}$, which is focused to a 1.5 - $\mu \mathrm{m}$ spot by a NIR $\times 50$ microscope objective. The train of laser pulses is actively locked to the RF signal exciting the SAW to ensure optical excitation at an arbitrary but well-defined time during the acoustic cycle. ${ }^{51}$ The emission from the sample is dispersed by a $0.75-\mathrm{m}$ imaging grating monochromator. A fast $(<50 \mathrm{ps}$ rise time) Si single-photon avalanche detector is used for time-resolved singlechannel detection of the spectral modulation of the optical modes ${ }^{52}$. Details of the phase-locked excitation scheme and additional data on single-cavity measurements are discussed in Supplementary Note 2 and Supplementary Fig. 5.

Sample structure. We start by fabricating the $\mathrm{PhC}$ membranes from a semiconductor heterostructure grown by molecular beam epitaxy (MBE). This heterostructure consists of a 170-nm GaAs layer with self-assembled InGaAs QDs at its centre, on top of a 725-nm-thick $\mathrm{Al}_{0.8} \mathrm{Ga}_{0.2} \mathrm{As}$ sacrificial layer. The PM structure is defined by electron beam lithography and transferred into the heterostructure by inductively coupled plasma reactive ion etching (ICP-RIE) etching. In a wet chemical etching step using hydrofluoric acid, we removed the sacrificial layer to release a fully suspended membrane. The PMs are deliberately designed to be offresonant with the QD emission to achieve a sufficiently long decay time of the cavity emission. The cavity resonance is detuned by several $\mathrm{meV}$ from the QD emission band. Thus, no Purcell-enhanced spontaneous emission with fast radiative rates occurs ${ }^{53}$. In turn, the cavity mode emission arises from phonon- or Coulomb-assisted feeding mechanisms on a $\sim 1$-ns timescale ${ }^{54-56}$. These long timescales set the experimental time window over which the cavity mode can be detected using time-resolved photoluminescence spectroscopy. Additional data of time-resolved experiments of the cavity emission are shown in Supplementary Fig. 5d.

IDTs were defined using electron beam lithography and metallized with $5 \mathrm{~nm}$ Ti and $50 \mathrm{~nm} \mathrm{Al}$ in a lift-off process. The finger period is $3.83 \mu \mathrm{m}$, resulting in a resonance frequency of $800 \mathrm{MHz}$ at the measurement temperature of $5 \mathrm{~K}$. The IDTs consist of 80 pairs of $185-\mu \mathrm{m}$-long fingers and are located at a distance of $800 \mu \mathrm{m}$ from the array of PhCs. The PhCs are arranged in a staggered pattern, that is, offset in the direction perpendicular to the SAW propagation. This arrangement ensures comparable SAW coupling to each $\mathrm{PhC}$ device. A schematic of this layout is included as Supplementary Fig. 6.

Coupled mode model. We treat the PM as a model system of two coupled cavities The single-cavity modes $M_{1,2}$ have the complex amplitudes $a_{1,2}$ and the frequencies $\omega_{1,2}$. In the presence of finite coupling and the absence of dissipation, the time evolution is given by

$$
\frac{\mathrm{d} a_{1}}{\mathrm{~d} t}=i \omega_{1} a_{1}+i \frac{J}{2} a_{2}
$$

and

$$
\frac{\mathrm{d} a_{2}}{\mathrm{~d} t}=i \omega_{2} a_{2}+i \frac{I}{2} a_{1}
$$

with a real coupling constant $J$.

The resulting normal modes have the frequencies given by equation (1) $\omega_{ \pm}=$ $\omega_{0} \pm \frac{1}{2} \sqrt{\Delta^{2}+J^{2}}$ with centre frequency $\omega_{0}=\frac{1}{2}\left(\omega_{1}+\omega_{2}\right)$ and detuning $\Delta=\omega_{2}-\omega_{1}$. We note that since $J>0$, the low-frequency mode, $\omega_{-}$, and the highfrequency mode, $\omega_{+}$, correspond to symmetric and antisymmetric superpositions of the uncoupled, single-cavity modes, respectively.

\section{References}

1. Notomi, M. Manipulating light with strongly modulated photonic crystals. Rep. Prog. Phys. 73, 096501 (2010).

2. O’Brien, J. L., Furusawa, A. \& Vuckovic, J. Photonic quantum technologies Nat. Photon. 3, 687-695 (2009).

3. Faraon, A. et al. Integrated quantum optical networks based on quantum dots and photonic crystals. New. J. Phys. 13, 055025 (2011).

4. Volz, T. et al. Ultrafast all-optical switching by single photons. Nat. Photon. 6, 605-609 (2012).

5. Hartmann, M. J., Brandao, F. \& Plenio, M. B. Strongly interacting polaritons in coupled arrays of cavities. Nat. Phys. 2, 849-855 (2006).

6. Yang, X., Yu, M., Kwong, D.-L. \& Wong, C. W. All-optical analog to electromagnetically induced transparency in multiple coupled photonic crystal cavities. Phys. Rev. Lett. 102, 173902 (2009).

7. Grillet, C. et al. Reconfigurable photonic crystal circuits. Laser Photonics. Rev. 4, 192-204 (2010).

8. Shevchenko, S. N., Ashhab, S. \& Nori, F. Landau-Zener-Stückelberg interferometry. Phys. Rep. 492, 1-30 (2010).

9. Blattmann, R., Krenner, H. J., Kohler, S. \& Hänggi, P. Entanglement creation in a quantum-dot-nanocavity system by Fourier-synthesized acoustic pulses. Phys. Rev. A 89, 012327 (2014). 
10. Vlasov, Y. A., O’Boyle, M., Hamann, H. F. \& McNab, S. J. Active control of slow light on a chip with photonic crystal waveguides. Nature 438, 65-69 (2005).

11. Tanabe, T., Notomi, M., Kuramochi, E., Shinya, A. \& Taniyama, H. Trapping and delaying photons for one nanosecond in an ultrasmall high-Q photonic-crystal nanocavity. Nat. Photon. 1, 49-52 (2007).

12. Laucht, A. et al. Electrical control of spontaneous emission and strong coupling for a single quantum dot. New. J. Phys. 11, 023034 (2009).

13. Jin, C.-Y. et al. Ultrafast non-local control of spontaneous emission. Nat. Nanotechnol. 9, 886-890 (2014).

14. Pagliano, F. et al. Dynamically controlling the emission of single excitons in photonic crystal cavities. Nat. Commun. 5, 5786 (2014).

15. Bayer, M. et al. Optical modes in photonic molecules. Phys. Rev. Lett. 81, 2582-2585 (1998).

16. Cai, T., Bose, R., Solomon, G. S. \& Waks, E. Controlled coupling of photonic crystal cavities using photochromic tuning. Appl. Phys. Lett. 102, 141118 (2013).

17. Chalcraft, A. R. A. et al. Mode structure of coupled L3 photonic crystal cavities. Opt. Express 19, 5670-5675 (2011).

18. Fuhrmann, D. A. et al. Dynamic modulation of photonic crystal nanocavities using gigahertz acoustic phonons. Nat. Photon. 5, 605-609 (2011).

19. de Lima, M. M. \& Santos, P. V. Modulation of photonic structures by surface acoustic waves. Rep. Prog. Phys. 68, 1639-1701 (2005).

20. de Lima, M. M., van der Poel, M., Santos, P. V. \& Hvam, J. M. Phonon-induced polariton superlattices. Phys. Rev. Lett. 97, 045501 (2006).

21. Ruppert, C. et al. Surface acoustic wave mediated coupling of free-space radiation into surface plasmon polaritons on plain metal films. Phys. Rev. B 82, 081416 (R) (2010).

22. Schiefele, J., Pedros, J., Sols, F., Calle, F. \& Guinea, F. Coupling light into graphene plasmons through surface acoustic waves. Phys. Rev. Lett. 111, 237405 (2013).

23. Ruppert, C. et al. Radio frequency electromechanical control over a surface plasmon polariton coupler. ACS Photon. 1, 91-95 (2014).

24. Beck, M. et al. Acousto-optical multiple interference switches. Appl. Phys. Lett. 91, 061118 (2007).

25. Tadesse, S. A. \& Li, M. Sub-optical wavelength acoustic wave modulation of integrated photonic resonators at microwave frequencies. Nat. Commun. 5, $5402(2014)$

26. Gell, J. R. et al. Modulation of single quantum dot energy levels by a surface-acoustic-wave. Appl. Phys. Lett. 93, 081115 (2008).

27. Metcalfe, M., Carr, S. M., Muller, A., Solomon, G. S. \& Lawall, J. Resolved sideband emission of InAs/GaAs quantum dots strained by surface acoustic waves. Phys. Rev. Lett. 105, 037401 (2010).

28. Völk, S. et al. Enhanced sequential carrier capture into individual quantum dots and quantum posts controlled by surface acoustic waves. Nano Lett. 10, 3399-3407 (2010).

29. Weiß, M. et al. Dynamic acoustic control of individual optically active quantum dot-like emission centers in heterostructure nanowires. Nano Lett. 14, 2256-2264 (2014)

30. Li, H. \& Li, M. Optomechanical photon shuttling between photonic cavities. Nat. Nanotechnol. 9, 913-919 (2014).

31. Sun, X., Zhang, X., Poot, M., Xiong, C. \& Tang, H. X. A superhigh-frequency optoelectromechanical system based on a slotted photonic crystal cavity. Appl. Phys. Lett. 101, 221116 (2012)

32. Sato, Y. et al. Strong coupling between distant photonic nanocavities and its dynamic control. Nat. Photon. 6, 56-61 (2012).

33. Bose, R., Cai, T., Choudhury, K. R., Solomon, G. S. \& Waks, E. All-optical coherent control of vacuum Rabi oscillations. Nat. Photon. 8, 858-864 (2014).

34. Schülein, F. J. R. et al. Fourier-synthesis of radio frequency nanomechanical pulses with different shapes. Nat. Nanotechnol. 10, 512-516 (2015).

35. Gavartin, E. et al. Optomechanical coupling in a two-dimensional photonic crystal defect cavity. Phys. Rev. Lett. 106, 203902 (2011).

36. Eichenfield, M., Chan, J., Camacho, R. M., Vahala, K. J. \& Painter, O. Optomechanical crystals. Nature 462, 78-82 (2009).

37. Akahane, Y., Asano, T., Song, B.-S. \& Noda, S. High-Q photonic nanocavity in a two-dimensional photonic crystal. Nature 425, 944-947 (2003).

38. Liew, T. C. H. \& Savona, V. Single photons from coupled quantum modes. Phys. Rev. Lett. 104, 183601 (2010).

39. Cirac, J. I., Zoller, P., Kimble, H. J. \& Mabuchi, H. Quantum state transfer and entanglement distribution among distant nodes in a quantum network. Phys. Rev. Lett. 78, 3221-3224 (1997).

40. Vasco, J. P., Guimarães, P. S. S. \& Gerace, D. Long-distance radiative coupling between quantum dots in photonic crystal dimers. Phys. Rev. B 90, 155436 (2014).

41. Stotz, J. A. H., Hey, R., Santos, P. V. \& Ploog, K. H. Coherent spin transport through dynamic quantum dots. Nat. Mater. 4, 585-588 (2005).
42. Notomi, M., Kuramochi, E. \& Tanabe, T. Large-scale arrays of ultrahigh-Q coupled nanocavities. Nat. Photon. 2, 741-747 (2008).

43. Liew, T. H. C. \& Savona, V. Multimode entanglement in coupled cavity arrays. New J. Phys. 15, 025015 (2013).

44. Gersen, H. et al. Direct observation of bloch harmonics and negative phase velocity in photonic crystal waveguides. Phys. Rev. Lett. 94, 123901 (2005).

45. Batista, P. D. et al. $\mathrm{ZnO} / \mathrm{SiO}_{2}$ microcavity modulator on silicon. Appl. Phys. Lett. 92, 133502 (2008).

46. Büyükköse, S. et al. Ultrahigh-frequency surface acoustic wave transducers on $\mathrm{ZnO} / \mathrm{SiO}_{2} / \mathrm{Si}$ using nanoimprint lithography. Nanotechnology 23, 315303 (2012).

47. Gustafsson, M. V. et al. Propagating phonons coupled to a superconducting qubit. Science 346, 207-211 (2014).

48. Eichenfield, M., Chan, J., Safavi-Naeini, A. H., Vahala, K. J. \& Painter, O. Modeling dispersive coupling and losses of localized optical and mechanical modes in optomechanical crystals. Opt. Express 17, 20078-20098 (2009).

49. Safavi-Naeini, A. H. et al. Two-dimensional phononic-photonic band gap optomechanical crystal cavity. Phys. Rev. Lett. 112, 153603 (2014).

50. Ludwig, M. \& Marquart, F. Quantum many-body dynamics in optomechanical arrays. Phys. Rev. Lett. 111, 073603 (2013).

51. Völk, S. et al. Direct observation of dynamic surface acoustic wave controlled carrier injection into single quantum posts using phase-resolved optical spectroscopy. Appl. Phys. Lett. 98, 023109 (2011).

52. Schülein, F. J. R. et al. Acoustically regulated carrier injection into a single optically active quantum dot. Phys. Rev. B 88, 085307 (2013).

53. Kress, A. et al. Manipulation of the spontaneous emission dynamics of quantum dots in two- dimensional photonic crystals. Phys. Rev. B 71, 241304 (R) (2005).

54. Winger, W. et al. Explanation of photon correlations in the far-off-resonance optical emission from a quantum-dot-cavity system. Phys. Rev. Lett. 103, 207403 (2009)

55. Laucht, A. et al. Temporal monitoring of nonresonant feeding of semiconductor nanocavity modes by quantum dot multiexciton transitions. Phys. Rev. B 81, 241302(R) (2010).

56. Florian, M., Gartner, P., Steinhoff, A., Gies, C. \& Jahnke, F. Coulomb-assisted cavity feeding in nonresonant optical emission from a quantum dot. Phys. Rev. B 89, 161302(R) (2014).

\section{Acknowledgements}

This work was supported by the Deutsche Forschungsgemeinschaft (DFG) via the Emmy Noether Programme (KR 3790/2-1), Sonderforschungsbereich 631 and the Cluster of Excellence Nanosystems Initiative Munich (NIM). K.M. acknowledges support by the Alexander von Humboldt Foundation.

\section{Author contributions}

H.J.K. and S.K. designed the study. S.K. built the experimental set-up and performed the experiments. S.K., T.R. and S.L. designed, fabricated and characterized the devices, and performed 3D-FDTD simulations. K.M. fabricated and characterized the MBE material S.K. and H.J.K. performed the data analysis and modelling. All authors discussed the results. S.K. and H.J.K. wrote the manuscript with contributions from all other authors H.J.K. proposed, initiated and coordinated research. H.J.K., M.K., J.J.F. and A.W. inspired and supervised the project.

\section{Additional information}

Supplementary Information accompanies this paper at http://www.nature.com/ naturecommunications

Competing financial interests: The authors declare no competing financial interests

Reprints and permission information is available online at http://npg.nature.com/ reprintsandpermissions/

How to cite this article: Kapfinger, S. et al. Dynamic acousto-optic control of a strongly coupled photonic molecule. Nat. Commun. 6:8540 doi: 10.1038/ncomms9540 (2015)

This work is licensed under a Creative Commons Attribution 4.0 International License. The images or other third party material in this article are included in the article's Creative Commons license, unless indicated otherwise in the credit line; if the material is not included under the Creative Commons license, users will need to obtain permission from the license holder to reproduce the material. To view a copy of this license, visit http://creativecommons.org/licenses/by/4.0/ 